\title{
Antiretroviral agents and acid-base balance at delivery of the neonate
}

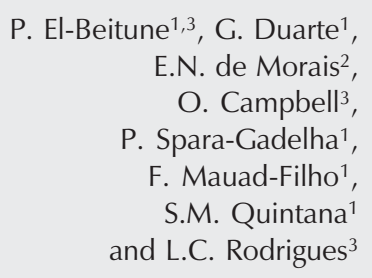

\author{
${ }^{1}$ Departamento de Ginecologia e Obstetrícia, Faculdade de Medicina de Ribeirão \\ Preto, Universidade de São Paulo, Ribeirão Preto, SP, Brasil \\ ${ }^{2}$ Departamento de Ginecologia e Obstetrícia, Centro de Ciências de Saúde, \\ Universidade Federal de Santa Maria, Santa Maria, RS, Brasil \\ ${ }^{3}$ Infectious Disease Epidemiology Unit, London School of Hygiene and Tropical \\ Medicine, London, UK
}

\section{Correspondence \\ P. El-Beitune \\ Maternidade Mário Totta \\ Complexo Hospitalar Santa Casa \\ Rua Prof. Annes Dias, 285, 10 andar \\ 90020-090 Porto Alegre, RS \\ Brasil \\ Fax: +55-51-3372-1401 \\ E-mail: pbeitune@yahoo.com.br \\ Research supported by FAPESP \\ (No. 01/08450-8).}

Received June 27, 2006

Accepted May 2, 2007

\begin{abstract}
Limited evidence is available regarding antiretroviral (ARV) safety for uninfected infants exposed to these drugs in utero. Our objective was to determine if ARV administered to pregnant women is associated with decreasing umbilical arterial $\mathrm{pH}$ and base excess in uninfected infants. A prospective study was conducted on 57 neonates divided into three groups: ZDV group, born to mothers taking zidovudine $(\mathrm{N}$ $=20$ ), triple therapy (TT) group, born to mothers taking zidovudine + lamivudine + nelfinavir $(\mathrm{N}=25)$, and control group $(\mathrm{N}=12)$, born to uninfected mothers. Umbilical cord blood was used to determine umbilical artery gases. A test was performed to calculate the sample by comparing means by the unpaired one-tailed $t$-test, with $\alpha=0.05$ and $\beta=20 \%$, indicating the need for a sample of 18 newborn infants for the study groups to detect differences higher than $20 \%$. The control and ARV groups were similar in gestational age, birth weight, and Apgar scores. Values of $\mathrm{pH}, \mathrm{pCO}_{2}$, bicarbonate, and base excess in cord arterial blood obtained at delivery from the newborns exposed to TT were $7.23,43.2 \mathrm{mmHg}, 19.5 \mathrm{mEq} / \mathrm{L}$, and $-8.5 \mathrm{nmol} / \mathrm{L}$, respectively, with no significant difference compared to the control and ZDV groups. We conclude that intrauterine exposure to ARV is not associated with a pathological decrease in umbilical arterial $\mathrm{pH}$ or base excess. While our data are reassuring, follow-up is still limited and needs to be continued into adulthood because of the possible potential for adverse effects of triple antiretroviral agents.
\end{abstract}

Pregnant women are increasingly being treated with multiple antiretroviral (ARV) drugs to improve maternal health and to reduce the risk of vertical transmission of HIV infection. Nucleoside reverse transcriptase inhibitors cross the placenta and reach a variable newborn/mother drug ratio, i.e., ap-
Key words - HIV

- Infant toxicity

- Pregnancy

- Umbilical arterial pH

- Apgar scores proximately 0.85 for zidovudine (ZDV) and 1.0 for lamivudine. Although this maternalfetal transfer is beneficial for the prevention of the vertical transmission of HIV-1, it may expose the infant to the risk of adverse effects $(1,2)$.

Although this subject is controversial, 
prophylaxis with ZDV has been implicated in changes in the neurological and cognitive development of children exposed to HIV but not infected with the virus (3). Limited evidence is available regarding ARV safety for uninfected infants exposed to these drugs in utero. Most of the studies that have addressed the intrauterine well-being of these infants have determined a perinatal prognosis based on Apgar scores and fetal and neonatal death $(4,5)$. In addition, umbilical artery blood $\mathrm{pH}$ and gas analysis are increasingly recognized as the most reliable indicators of fetal oxygenation and acid-base condition at birth $(6,7)$. Therefore, our objective was to evaluate the effect of two different intrauterine exposures, ZDV alone or triple antiretroviral treatment with ZDV, lamivudine (3TC) and nelfinavir (NFV) on the umbilical artery blood $\mathrm{pH}$ and gas analysis at birth in HIV-exposed children.

We carried out a prospective study on 45 HIV-1-infected women and 12 uninfected pregnant women with singleton gestations. Only HIV-infected patients who had not been treated previously with ARV were selected for the study. The HIV-1-infected women were divided into two groups named ZDV group and triple treatment (TT) group. The ZDV group consisted of 20 pregnant women who fulfilled the requirements for the prophylactic use of ZDV $\left(\mathrm{CD}_{4}>500\right.$ cells $/ \mathrm{mm}^{3}$ and viral load $<1000$ copies $/ \mathrm{mL}$ ). The TT group consisted of 25 pregnant women with a clinical and laboratory indication $\left(\mathrm{CD}_{4}<500\right.$ cells $\left./ \mathrm{mm}^{3}\right)$ for triple antiretroviral treatment $(\mathrm{ZDV}+3 \mathrm{TC}+\mathrm{NFV})$ according to the criteria established by the Perinatal HIV Guidelines Working Group Members regarding ARV treatment of pregnant women (2).

The ARV agents recommended since the 14th week of pregnancy were ZDV, $300 \mathrm{mg} /$ dose, twice a day for the ZDV group, and $300 \mathrm{mg}$ ZDV, $150 \mathrm{mg}$ 3TC, and $1250 \mathrm{mg}$ NFV in two daily doses for the TT group. The present study was approved by the Re- search Ethics Committee of the Institution, Hospital das Clínicas da Faculdade de Medicina de Ribeirão Preto, Universidade de São Paulo, Ribeirão Preto, SP, Brazil, and written informed consent to participate was obtained from each subject.

To evaluate factors potentially associated with umbilical arterial blood $\mathrm{pH}$ abnormalities from birth, we adjusted analyses for gestational age at birth, mode of delivery, alcohol use, illicit drug use during gestation, and tobacco use. Pregnant women with hypertension, diabetes mellitus, seizures, stillbirth, or macrosomia in their previous pregnancies, and pathological fetal heart rate tracings and women who did not comply with the use of ARV drugs or used them irregularly and also infants presenting congenital infections or malformations were excluded. Biochemical evaluation was not concluded in two ZDV group and three TT group infants due to concomitance of exclusion criteria.

Blood samples were drawn from a doubly clamped cord segment into 3-mL plastic syringes flushed with a $1000-\mathrm{U} / \mathrm{mL}$ heparin solution. Measurements were performed within 15 min after delivery. Arterial blood gases were determined by an automated method using the Rapid Lab-860 system of Bayer (Tarrytown, NY, USA).

Since we expected that the use of ARV drugs during pregnancy would elevate $\mathrm{pCO}_{2}$ and reduce $\mathrm{pH}$ and base excess compared to controls (12 newborn infants), a test was performed to calculate the sample by comparing means by the unpaired one-tailed $t$ test, with $\alpha=0.05$ and $\beta=20 \%$, indicating the need for a sample of 18 newborn infants for the study groups, considering the interest in detecting pathologic data of $\mathrm{pCO}_{2}$ of about $65 \mathrm{mmHg}, \mathrm{pH} 7.10$, and base excess higher than $12 \mathrm{nmol} / \mathrm{L}$. The GraphPad StatMate 1.01 software was used for these calculations.

The variability of the acid-base balance in cord arterial blood was calculated on the 
basis of the median and interquartile variation (1st and 3rd quartiles, respectively). The nonparametric chi-square, Mann-Whitney and Kruskal-Wallis tests were used, with the level of significance set at $\mathrm{P}<0.05$. All analyses were performed using the SPSS 10.0 software.

Median maternal age was 22.5 years, with an interquartile (IQ) variation of 6 years in the control group, 24 years (IQ of 7 years) in the ZDV group, and 27 years (IQ of 6 years) in the TT group, with no significant difference in these variables between groups (Kruskal-Wallis test, $\mathrm{P}=0.13$ ). With respect to race (white and non-white), 83, 50, and $68 \%$ of the women in the control, ZDV and TT groups were white, respectively (chisquare test, $\mathrm{P}=0.14)$. Smoking habits also were not significantly different, with 91,60 , and $80 \%$ of the subjects in the control, ZDV and TT groups being non-smokers, respectively (chi-square test, $\mathrm{P}=0.10$ ). Alcohol drinking also did not differ significantly between groups (chi-square test, $\mathrm{P}=0.14$ ).

Median gestational age at delivery was 39 weeks for the control group, 38.1 weeks for the ZDV group and 38.5 weeks for the TT group (Kruskal-Wallis test, $\mathrm{P}=0.57$ ). Median infant weight was 3250, 3080, and $3100 \mathrm{~g}$ for the control, ZDV and TT groups, respectively (Kruskal-Wallis test, $\mathrm{P}=0.447$ ). Analysis of these variables, of the Apgar score and of adequacy of anthropometric classification did not indicate any signifi- cant differences among the newborns of the various groups (chi-square test, $\mathrm{P}=0.59$ ). Cesarean section was performed in 16.7, 45.0, and $36.0 \%$ of the control, ZDV and TT groups, respectively (chi-square test, $\mathrm{P}=$ $0.26)$.

Table 1 presents the values of $\mathrm{pH}, \mathrm{pCO}_{2}$, bicarbonate, and base excess in cord arterial blood from the newborn at the time of delivery. No significant differences were observed between cases and controls. Even though the results of the present series support the safety of the use of two schemes of ARV therapy during pregnancy, i.e., ZDV prophylaxis and combination of ARV agents, a limitation exists, based on the power of the study reported here to detect an effect of lower magnitude among groups.

In the present patient series, no difference in gestational age or in newborn birth weight was observed, nor did the 1st and 5th min Apgar scores differ between the three groups studied. These data are similar to those obtained in other studies and metaanalyses which demonstrated that the gestational and immediate neonatal prognosis were not impaired among pregnant women exclusively taking ZDV or taking drug combinations containing or not protease inhibitors during the prenatal period (8-11).

Despite the vast literature available about the potentiation of the adverse effects of ARV in adults $(2,11,12)$, there are no prospective studies emphasizing the effect of

\begin{tabular}{|c|c|c|c|c|}
\hline Group & $\mathrm{pH}$ & $\mathrm{pCO}_{2}(\mathrm{mmHg})$ & $\mathrm{HCO}_{3}(\mathrm{mEq} / \mathrm{L})$ & $\mathrm{BE}(\mathrm{nmol} / \mathrm{L})$ \\
\hline Control $(N=12)$ & $7.25(7.20-7.28)$ & $40.0(31.9-48.9)$ & $17.7(15.1-18.9)$ & $-10.3(-10.9$ to -6.9$)$ \\
\hline $\mathrm{ZDV}(\mathrm{N}=18)$ & $7.22(7.14-7.26)$ & $47.3(41.9-51.9)$ & $18.3(16.9-20.3)$ & $-8.8(-11.6$ to -6.9$)$ \\
\hline $\mathrm{TT}(\mathrm{N}=22)$ & $7.23(7.15-7.30)$ & $43.2(40.6-46.6)$ & $19.5(17.3-20.7)$ & $-8.5(-12.6$ to -5.8$)$ \\
\hline$P$ & 0.24 & 0.11 & 0.13 & 0.72 \\
\hline
\end{tabular}

Data are reported as medians and 1 st and 3rd quartiles. Control = uninfected pregnant women with no antiretroviral agents; ZDV = $300 \mathrm{mg}$ zidovudine twice a day; TT = triple therapy with $300 \mathrm{mg}$ zidovudine +150 $\mathrm{mg}$ lamivudine $+1250 \mathrm{mg}$ nelfinavir twice a day. There were no statistically significant differences between groups (Kruskal-Wallis test). 
maternal ARV agent use on acid-base balance at delivery of the neonate, which objectively reflects the placental respiratory and metabolic conditions of the infant at birth, using these data as a basis for the clinical results observed $(7,13,14)$.

The $\mathrm{pH}$ value observed was 7.25 for the control group, with no significant differences compared to the cases. Similarly, placental respiratory and metabolic function did not differ between groups. Our clinical data agree with those reported for children exposed to HIV but not infected followed up in the multicenter PACTG 076 study for a mean period of 4.2 years (range: 3.2-5.6 years), in which no difference in neurological, cognitive or behavioral development was observed compared to the control group (15). In addition, in a meta-analysis conducted on a total of 2123 HIV-infected pregnant women who had received ARV therapy during the prenatal period (ZDV alone in 1590 , combined therapy without protease inhibitors in 396, and combined therapy with protease inhibitors in 137) and on 1143 women who did not receive ARV therapy during pregnancy, the use of ARV medications was also not associated with low Apgar scores or fetal death compared to untreated women or women taking ZDV alone (9).

In conclusion, no association was observed between the use of ARV drugs and pathological gas changes in umbilical cord arterial blood, with similar data being detected for the three groups regarding $\mathrm{pH}$, $\mathrm{pCO}_{2}$, base deficit, and bicarbonate. Our results suggest safety of the use of ARV drugs during pregnancy regarding fetal oxygenation and acid-base condition at birth. On the other hand, the "gold standard" is to conduct follow-up of children with intrauterine ARV drug exposure into adulthood because of the possible potential for adverse metabolic and hematological effects of a combined scheme with protease inhibitors $(16,17)$.

\section{References}

1. Mandelbrot L, Peytavin G, Firtion G, Farinotti R. Maternal-fetal transfer and amniotic fluid accumulation of lamivudine in human immunodeficiency virus-infected pregnant women. Am J Obstet Gynecol 2001; 184: 153-158.

2. Perinatal HIV Guidelines Working Group Members. Public Health Service Task Force Recommendation for Safety and Toxicity of Individual Antiretroviral Agents in Pregnancy. http://www.hivatis.org. Accessed May 15, 2006.

3. Carneiro M, Sanchez A, Maneiro P, Angelosante W, Perez C, Vallee M. Vertical HIV-1 transmission: prophylaxis and paediatric followup. Placenta 2001; 22 (Suppl A): S13-S18.

4. El Beitune P, Duarte G, Foss MC, Montenegro RM Jr, Quintana SM, Figueiro-Filho EA, et al. Effect of maternal use of antiretroviral agents on serum insulin levels of the newborn infant. Diabetes Care 2005; 28: 856-859.

5. El Beitune P, Duarte G, Machado AA, Quintana SM, Figueiro-Filho EA, Abduch R. Effect of antiretroviral drugs on maternal CD4 lymphocyte counts, HIV-1 RNA levels, and anthropometric parameters of their neonates. Clinics 2005; 60: 207-212.

6. Page FO, Martin JN, Palmer SM, Martin RW, Lucas JA, Meeks GR, et al. Correlation of neonatal acid-base status with Apgar scores and fetal heart rate tracings. Am J Obstet Gynecol 1986; 154: 13061311.

7. Low JA, Lindsay BG, Derrick EJ. Threshold of metabolic acidosis associated with newborn complications. Am J Obstet Gynecol 1997; 177: 1391-1394.

8. El Beitune P, Duarte G, Quintana SM, Figueiro-Filho EA, Marcolin $A C$, Abduch R. Antiretroviral therapy during pregnancy and early neonatal life: consequences for HIV-exposed, uninfected children. Braz J Infect Dis 2004; 8: 140-150.

9. Tuomala RE, Shapiro DE, Mofenson LM, Bryson Y, Culnane M, Hughes MD, et al. Antiretroviral therapy during pregnancy and the risk of an adverse outcome. N Engl J Med 2002; 346: 1863-1870.

10. Brocklehurst P, Wolmink J. Antiretroviral for reducing the risk of mother-to-child transmission of HIV infection. 2: CD003510. Cochrane Database Syst Rev; 2002.

11. Mofenson LM. U.S. Public Health Service Task Force recommendations for use of antiretroviral drugs in pregnant HIV-1-infected women for maternal health and interventions to reduce perinatal HIV-1 transmission in the United States. MMWR Recomm Rep 2002; 51 : 1-38.

12. El Beitune P, Duarte G, Foss MC, Montenegro RM Jr, Spara P, Quintana SM, et al. Effect of antiretroviral agents on carbohydrate metabolism in HIV-1 infected pregnant women. Diabetes Metab Res Rev 2006; 22: 59-63.

13. Winkler CL, Hauth JC, Tucker JM, Owen J, Brumfield CG. Neonatal complications at term as related to the degree of umbilical artery acidemia. Am J Obstet Gynecol 1991; 164: 637-641. 
14. Kitlinski ML, Kallen K, Marsal K, Olofsson P. Gestational age-dependent reference values for $\mathrm{pH}$ in umbilical cord arterial blood at term. Obstet Gynecol 2003; 102: 338-345.

15. Culnane M, Fowler M, Lee SS, McSherry G, Brady M, O'Donnell K, et al. Lack of long-term effects of in utero exposure to zidovudine among uninfected children born to HIV-infected women. Pediatric AIDS Clinical Trials Group Protocol 219/076 Teams. JAMA 1999; 281: 151-157.
16. El Beitune P, Duarte G. Antiretroviral agents during pregnancy: consequences on hematologic parameters in HIV-exposed, uninfected newborn infant. Eur J Obstet Gynecol Reprod Biol 2006; 128: 5963.

17. El Beitune P, Duarte G, Vannucchi H, Quintana SM, Figueiro-Filho $E A$, de Morais EN, et al. Serum vitamin A during pregnancy and effects on obstetrics and perinatal outcomes in HIV infected pregnant women. Arch Latinoam Nutr 2004; 54: 419-427. 